\title{
Boundary Behavior and Cesàro Means of Universal Taylor Series
}

\author{
Frédéric BAYART \\ Laboratoire Bordelais d'Analyse et de Géométrie \\ UMR 5467, Université Bordeaux 1 \\ 351 Cours de la Libération \\ F-33405 Talence cedex — France \\ Frederic.Bayart@math.u-bordeaux1.fr
}

Received: July 26, 2005

Accepted: January 9, 2006

\begin{abstract}
We study boundary properties of universal Taylor series. We prove that if $f$ is a universal Taylor series on the open unit disk, then there exists a residual subset $G$ of the unit circle such that $f$ is unbounded on all radii with endpoints in $G$. We also study the effect of summability methods on universal Taylor series. In particular, we show that a Taylor series is universal if and only if its Cesàro means are universal.

Key words: cluster set, universality, overconvergence, Bernstein's inequality

2000 Mathematics Subject Classification: Primary 30B40. Secondary 30B10, 30D40, 30E10, 40G05.
\end{abstract}

\section{Introduction}

A notion of universal Taylor series in the complex plane with respect to overconvergence was defined in the early 1970's independently by Luh [8] and Chui and Parnes [2]. Later on, Nestoridis [11] introduced a stronger notion of universality of Taylor series. In the present work, we deal with universal Taylor series in the sense of Nestoridis, and we are mainly concerned with their boundary behavior and their summability. Let us first recall some notations and definitions. We denote by $H(\mathbb{D})$ the set of holomorphic functions on the unit disk $\mathbb{D}$, endowed with the topology of uniform convergence on compact sets. For $f \in H(\mathbb{D}), S_{n}(f, \xi)(z)$ is the $n$-th partial sum of the Taylor series of $f$ with center at $\xi \in \mathbb{D}$. For $K$ a subset of $\mathbb{C}, K^{0}$ is the interior of $K$, and $K^{c}$ is its complement. $\bar{D}(w, r)$ stands for the closed disk with center $w \in \mathbb{C}$ and radius $r>0$. 
Definition 1.1. Let $\xi \in \mathbb{D}$. A holomorphic function $f \in H(\mathbb{D})$ belongs to the class $U(\mathbb{D}, \xi)$ provided that for every compact set $K \subset \mathbb{C}$ with $K \cap \mathbb{D}=\emptyset$ and $K^{c}$ being connected, and for every function $h: K \rightarrow \mathbb{C}$ continuous on $K$ and holomorphic in $K^{0}$, there exists a sequence $\left(\lambda_{n}\right)$ of natural numbers such that $S_{\lambda_{n}}(f, \xi)(z)$ converges to $h(z)$ uniformly on $K($ as $n \rightarrow+\infty)$.

In the particular case when $\xi=0$, this definition has the following interpretation: if $f$ belongs to $U(\mathbb{D}, 0)$, then its Taylor expansion has a very good behavior on any compact subset $K$ of $\mathbb{D}$, since it converges uniformly to $f$ on $K$ (as for any function $f \in H(\mathbb{D}))$. However, outside $\mathbb{D}$, and even on the unit circle $\mathbb{T}$, the sequence of partial sums of the Taylor series of $f$ has a very strange behavior (in fact, the worst possible behavior), since it may approximate any reasonable function on any reasonable set.

We are also concerned with universal Taylor series with respect to every center $\xi \in \mathbb{D}$.

Definition 1.2. A holomorphic function $f \in H(\mathbb{D})$ belongs to the class $U(\mathbb{D})$ provided that for every compact set $K \subset \mathbb{C}$ with $K \cap \mathbb{D}=\emptyset$ and $K^{c}$ being connected, and for every function $h: K \rightarrow \mathbb{C}$ continuous on $K$ and holomorphic in $K^{0}$, there exists a sequence $\left(\lambda_{n}\right)$ of natural numbers such that, for any compact set $N \subset \mathbb{D}$,

$$
\sup _{\xi \in N} \sup _{z \in K}\left|S_{\lambda_{n}}(f, \xi)(z)-h(z)\right| \rightarrow 0 \quad \text { as } \quad n \rightarrow+\infty \text {. }
$$

A suitable application of Baire's Theorem and Mergelyan's Theorem shows that $U(\mathbb{D}, \xi)$ and $U(\mathbb{D})$ are dense $G_{\delta}$ subsets of $H(\mathbb{D})$. Moreover, it is proved in [10] that $U(\mathbb{D})=U(\mathbb{D}, \xi)$ for any $\xi \in \mathbb{D}$. We mention that in $[10]$, it is proved that these results hold if we replace $\mathbb{D}$ by a simply connected domain $\Omega$ contained in some half-plane.

It is known that every function $f \in U(\mathbb{D})$ behaves quite badly near the boundary of $\mathbb{D}$. For instance, if $f \in U(\mathbb{D})$, then its Taylor expansion $S_{n}(f, \xi)(z)$ at $\xi \in \mathbb{D}$ is not $(C, k)$-summable for any $z \in \mathbb{T}$ and for any $k=1,2, \ldots$ (see $[7])$. On the contrary, for Abel summability, the situation is better. It was proved by D. Armitage and G. Costakis in [1] (see also [4] for a less strong conclusion) that if $E$ is any subset of the first category of the unit circle, if $\varepsilon:[0,1) \rightarrow(0,+\infty)$ is any continuous decreasing function and if $g$ is any holomorphic function in the open unit disk $\mathbb{D}$, then there exists $f \in U(\mathbb{D})$ such that

$$
|f(r z)-g(r z)|<\varepsilon(r) \quad \text { for any } \quad z \in E \text {. }
$$

In the same paper, a kind of negative counterpart is proved, namely that if $f \in U(\mathbb{D})$, then there exists a residual subset $G$ of $\mathbb{T}$ such that the set $\left\{f^{(n)}(r z)\right.$; $0<r<1\}$ is unbounded for every $z \in G$ and every positive integer $n$. As noted in [1], the behavior of the universal Taylor series itself along radii (case $n=0$ ) remains a "grey zone." In this paper, we address this question by showing that the above property remains true for $n=0$ (see Theorem 3.2 below). 
Recently, Melas and Nestoridis have studied in [10] the existence of universal Taylor series with respect to summability methods. We restrict ourselves to $(C, k)$ summability for $k \geq 0$ an integer. If $f \in H(\mathbb{D})$ and $\xi \in \mathbb{D}, \sigma_{n}^{k}(f, \xi)(z)$ is the $(C, k)$-mean of order $n$ of the Taylor expansion of $f$ with center at $\xi$. Namely, for $S_{n}(f, \xi)(z)=\sum_{\nu=0}^{n} a_{\nu}(z-\xi)^{\nu}$, one has

$$
\sigma_{n}^{j}(f, \xi)(z)=\sum_{\nu=0}^{n} \frac{A_{n-\nu}^{j}}{A_{n}^{j}} a_{\nu}(z-\xi)^{\nu},
$$

where $A_{m}^{l}=\frac{(m+1) \cdots(m+l)}{l !}$. (We refer to [13] for standard properties of Cesàro means of series.)

A corollary of Theorem 1.4 of [10] is the following

Theorem 1.3. There exists a holomorphic function $f \in H(\mathbb{D})$ with the following property: given a compact set $K \subset \mathbb{C}, K \cap \mathbb{D}=\emptyset, K^{c}$ connected, and a function $h: K \rightarrow \mathbb{C}$ continuous on $K$ and holomorphic in $K^{0}$, there exists a sequence $\left(\lambda_{m}\right)$ of natural numbers such that for any $k \geq 0$ and for any compact set $N \subset \mathbb{D}$,

$$
\sup _{\xi \in N} \sup _{z \in K}\left|\sigma_{\lambda_{m}}^{k}(f, \xi)(z)-h(z)\right| \rightarrow 0 \quad \text { as } \quad m \rightarrow+\infty .
$$

In [10], the authors consider more general Cesàro means $(C, a), a \in(-1,+\infty)$.

Let us denote by $U_{\mathrm{Ces}}(\mathbb{D})$ the set of functions $f$ satisfying the conclusion of the previous theorem: $U_{\mathrm{Ces}}(\mathbb{D})$ is the set of universal Taylor series with respect to every center and every Cesàro mean. It is plain that $U_{\text {Ces }}(\mathbb{D}) \subset U(\mathbb{D})$. In this paper, we prove the converse inclusion holds.

The article is organized as follows: in section 2, we recall some definitions and some results on Ostrowski gaps for Taylor series. Ostrowski gaps are indeed our main tool to prove our results. Next, section 3 is devoted to the study of the radial limits of universal Taylor series, whereas in section 4 , we are interested in summability methods for universal Taylor series.

\section{Ostrowski gaps of universal Taylor series}

Ostrowski gaps were successfully used in $[5,9,10]$ to obtain certain properties of universal Taylor series with respect to overconvergence. We first recall the definition of Ostrowski gaps.

Definition 2.1. Let $\sum_{\nu=0}^{+\infty} a_{\nu}(z-\xi)^{\nu}$ be a power series with convergence radius $r \in(0,+\infty)$. We say that it has Ostrowski gaps $\left(p_{m}, q_{m}\right)$ if $\left(p_{m}\right)$ and $\left(q_{m}\right)$ are sequences of natural numbers with

(i) $p_{1}<q_{1} \leq p_{2}<q_{2} \leq \cdots$ and $\lim _{m \rightarrow+\infty} \frac{q_{m}}{p_{m}}=\infty$.

(ii) For $I=\bigcup_{m=1}^{\infty}\left\{p_{m}+1, \ldots, q_{m}\right\}$, we have $\lim _{\nu \in I}\left|a_{\nu}\right|^{1 / \nu}=0$. 
We shall use the two following results concerning Ostrowski gaps.

Lemma 2.2 ([9, Theorem 1]). Let $f \in H(\mathbb{D}), \xi_{0} \in \mathbb{D}$. Suppose that the Taylor series of $f$ at center $\xi_{0}$ has Ostrowski gaps $\left(p_{m}, q_{m}\right)$. Then the difference $S_{p_{m}}(f, \xi)(z)-$ $S_{p_{m}}\left(f, \xi_{0}\right)(z)$ converges to zero (as $m \rightarrow \infty$ ) uniformly on compact sets of $\mathbb{D} \times \mathbb{C}$ $(\xi \in \mathbb{D}, z \in \mathbb{C})$.

Lemma 2.3 ([10, Theorem 9.1]). Let $\xi_{0} \in \mathbb{D}, f \in U\left(\mathbb{D}, \xi_{0}\right), K \subset \mathbb{C}$ be compact, such that $K \cap \mathbb{D}=\emptyset$ and $K^{c}$ is connected. Also, let $h: K \rightarrow \mathbb{C}$ be a continuous function on $K$ which is holomorphic in $K^{0}$. Then there exist two sequences of natural numbers $\left(p_{m}\right),\left(q_{m}\right)$ such that

(i) The Taylor series of $f$ at $\xi_{0}$ has Ostrowski-gaps $\left(p_{m}, q_{m}\right)$.

(ii) For any compact set $N \subset \mathbb{D}$, $\sup _{\xi \in N} \sup _{z \in K}\left|S_{p_{m}}(f, \xi)(z)-h(z)\right| \rightarrow 0$, as $m \rightarrow+\infty$.

As a corollary of this lemma, one gets $U(\mathbb{D})=U\left(\mathbb{D}, \xi_{0}\right)$ for any $\xi_{0} \in \mathbb{D}$. In our setting, two supplementary results about Ostrowski turns out to be useful.

Lemma 2.4. Let $f \in H(\mathbb{D})$, and suppose that the Taylor expansion of $f$ about $\xi_{0} \in \mathbb{D}$ has Ostrowski gaps $\left(p_{m}, q_{m}\right)$. Then for every sequence $\left(r_{m}\right)$ with $q_{m} / r_{m} \rightarrow+\infty$ and $r_{m} \geq p_{m}$, the difference $S_{r_{m}}(f, \xi)(z)-S_{p_{m}}(f, \xi)(z)$ converges uniformly to zero (as $m \rightarrow+\infty)$ on compact sets of $\mathbb{D} \times \mathbb{C}(\xi \in \mathbb{D}, z \in \mathbb{C})$.

Proof. It is sufficient to notice that

$$
\begin{aligned}
\mid S_{p_{m}}(f, \xi)(z)- & S_{r_{m}}(f, \xi)(z)|\leq| S_{p_{m}}(f, \xi)(z)-S_{p_{m}}\left(f, \xi_{0}\right)(z) \mid \\
& +\left|S_{p_{m}}\left(f, \xi_{0}\right)(z)-S_{r_{m}}\left(f, \xi_{0}\right)(z)\right|+\left|S_{r_{m}}\left(f, \xi_{0}\right)(z)-S_{r_{m}}(f, \xi)(z)\right| .
\end{aligned}
$$

Observe that the Taylor expansion of $f$ about $\xi_{0}$ has Ostrowski gaps $\left(p_{m}, q_{m}\right)$ as well as $\left(r_{m}, q_{m}\right)$. By Lemma $2.2, S_{p_{m}}(f, \xi)(z)-S_{p_{m}}\left(f, \xi_{0}\right)(z)$ and $S_{r_{m}}\left(f, \xi_{0}\right)(z)-S_{r_{m}}(f, \xi)(z)$ converge uniformly to zero on compact sets of $\mathbb{D} \times \mathbb{C}$. The definition of Ostrowski gaps now implies that $S_{p_{m}}\left(f, \xi_{0}\right)(z)-S_{r_{m}}\left(f, \xi_{0}\right)(z)$ tends to 0 uniformly on compact sets of $\mathbb{C}$.

The last lemma that we need is similar to Lemma 2.3, except that we are now dealing with Cesàro means instead of partial sums. So let us introduce the following definitions.

Definition 2.5. Let $\xi_{0} \in \mathbb{D}, j \geq 0$ and $f \in H(\mathbb{D})$.

- We say that $f$ belongs to $U_{\operatorname{Ces}(j)}\left(\mathbb{D}, \xi_{0}\right)$ provided that for every compact set $K \subset \mathbb{C}, K \cap \mathbb{D}=\emptyset, K^{c}$ connected, and every function $h: K \rightarrow \mathbb{C}$ continuous on $K$ and holomorphic in $K^{0}$, there exists a sequence $\left(\lambda_{m}\right)$ of natural numbers such that

$$
\sup _{z \in K}\left|\sigma_{\lambda_{m}}^{j}\left(f, \xi_{0}\right)(z)-h(z)\right| \rightarrow 0 \quad \text { as } \quad m \rightarrow+\infty
$$


- We say that $f$ belongs to $U_{\operatorname{Ces}(j)}(\mathbb{D})$ provided that for every compact set $K \subset \mathbb{C}$, $K \cap \mathbb{D}=\emptyset, K^{c}$ connected, and every function $h: K \rightarrow \mathbb{C}$ continuous on $K$ and holomorphic in $K^{0}$, there exists a sequence $\left(\lambda_{m}\right)$ of natural numbers such that, for every compact set $N \subset \mathbb{D}$,

$$
\sup _{\xi \in N} \sup _{z \in K}\left|\sigma_{\lambda_{m}}^{j}(f, \xi)(z)-h(z)\right| \rightarrow 0 \quad \text { as } \quad m \rightarrow+\infty .
$$

- We say that $f$ belongs to $U_{\text {Ces }}\left(\mathbb{D}, \xi_{0}\right)$ provided that for every compact set $K \subset \mathbb{C}$, $K \cap \mathbb{D}=\emptyset, K^{c}$ connected, and every function $h: K \rightarrow \mathbb{C}$ continuous on $K$ and holomorphic in $K^{0}$, there exists a sequence $\left(\lambda_{m}\right)$ of natural numbers such that, for every $k \geq 0$,

$$
\sup _{z \in K}\left|\sigma_{\lambda_{m}}^{k}\left(f, \xi_{0}\right)(z)-h(z)\right| \rightarrow 0 \quad \text { as } \quad m \rightarrow+\infty .
$$

- We say that $f$ belongs to $U_{\text {Ces }}(\mathbb{D})$ provided that for every compact set $K \subset \mathbb{C}$, $K \cap \mathbb{D}=\emptyset, K^{c}$ connected, and every function $h: K \rightarrow \mathbb{C}$ continuous on $K$ and holomorphic in $K^{0}$, there exists a sequence $\left(\lambda_{m}\right)$ of natural numbers such that, for every compact set $N \subset \mathbb{D}$ and for every $k \geq 0$,

$$
\sup _{\xi \in N} \sup _{z \in K}\left|\sigma_{\lambda_{m}}^{k}(f, \xi)(z)-h(z)\right| \rightarrow 0 \quad \text { as } \quad m \rightarrow+\infty .
$$

Lemma 2.6. Let $\xi \in \mathbb{D}$ and $f \in U_{C e s(j)}(\mathbb{D}, \xi)$. Let $K$ be any compact subset of $\mathbb{C} \backslash \mathbb{D}$ such that $\mathbb{C} \backslash K$ is connected, and let $h$ be any continuous function on $K$ and holomorphic inside $K^{0}$. Then there exists two sequences $\left(p_{m}\right),\left(q_{m}\right)$ such that the Taylor series of $f$ at $\xi$ has Ostrowski gaps $\left(p_{m}, q_{m}\right)$, and $\sigma_{q_{m}}^{j}(f, \xi)(z) \rightarrow h(z)$ uniformly on $K$.

Proof. The first part of the proof goes along the lines of the proof of step 1 of Theorem 3 in [5]. Let $C_{m}$ denote the closed disk $C_{m}:=\{z ;|z-(3 / 2) m| \leq m\}$. For large $m, K \cap C_{m}$ is empty. Hence, one may find a sequence $\left(q_{m}\right)$ of natural numbers with $q_{m+1} \geq m q_{m}$ such that $\sigma_{q_{m}}^{j}(f, \xi)(z)$ converges to $h(z)$ uniformly on $K$ and $\left|\sigma_{q_{m}}^{j}(f, \xi)(z)\right| \leq 1$ on $C_{m}$. Using the Bernstein-Walsh Lemma (see [12]), this yields

$$
\max _{|z|=m}\left|\sigma_{q_{m}}^{j}(f, \xi)(z)\right| \leq \max _{|z-(3 / 2) m| \leq(5 / 2) m}\left|\sigma_{q_{m}}^{j}(f, \xi)(z)\right| \leq\left(\frac{5}{2}\right)^{q_{m}} .
$$

Let us now write $S_{n}(f, \xi)(z)=\sum_{\nu=0}^{n} a_{\nu}(z-\xi)^{\nu}$ and

$$
\sigma_{n}^{j}(f, \xi)(z)=\sum_{\nu=0}^{n} \frac{A_{n-\nu}^{j}}{A_{n}^{j}} a_{\nu}(z-\xi)^{\nu} .
$$


It follows from Cauchy's formula that

$$
\left|a_{\nu} \frac{A_{q_{m}-\nu}^{j}}{A_{q_{m}}^{j}}\right|^{1 / \nu} \leq \frac{1}{m}\left(\frac{5}{2}\right)^{q_{m} / \nu} \quad \text { for all } \quad \nu \leq q_{m} .
$$

We set $p_{m}=\max \left(\left[\log \left(q_{m}+1\right)\right],\left[q_{m} / \log m\right]\right)+1$. For $\nu \in\left[p_{m}, q_{m}\right]$, we have

$$
\begin{aligned}
\left(\frac{A_{q_{m}-\nu}^{j}}{A_{q_{m}}^{j}}\right)^{1 / \nu} & \geq\left(1-\frac{\nu}{q_{m}+1}\right)^{j / \nu} \geq\left(1-\frac{q_{m}}{q_{m}+1}\right)^{j / \nu}=\left(\frac{1}{q_{m}+1}\right)^{j / \nu} \\
& \geq\left(\frac{1}{q_{m}+1}\right)^{\frac{j}{\log \left(q_{m}+1\right)}}=e^{-j} .
\end{aligned}
$$

Hence, we get for any $\nu \in\left[p_{m}, q_{m}\right]$

$$
\left|a_{\nu}\right|^{1 / \nu} \leq m^{-(1-\ln (5 / 2))} e^{-j}
$$

Therefore, the Taylor series of $f$ about $\xi$ has Ostrowski gaps $\left(p_{m}, q_{m}\right)$.

\section{Radial limits of universal Taylor series}

The aim of this section is to address the question of Armitage and Costakis:

Theorem 3.1. If $f \in U(\mathbb{D})$, then there exists a residual subset $G \subset \mathbb{T}$ such that the set $\left\{f^{(n)}(r z) ; 0<r<1\right\}$ is unbounded for every $z \in G$ and every $n \geq 0$.

Since the intersection of two residual subsets remains a residual subset, in view of Armitage and Costakis' result, it is enough to prove the following theorem.

Theorem 3.2. If $f \in U(\mathbb{D})$, then there exists a residual subset $G \subset \mathbb{T}$ such that $\{f(r z) ; 0<r<1\}$ is unbounded for every $z \in G$.

Proof. Our approach is based on $(C, 1)$-summability of the partial sums of certain Taylor series. So, we will need Rogosinski's formula in a form already used in the theory of universal Taylor series (see for instance [10, Lemma 2.7]).

Lemma 3.3. Let $\left(c_{\nu}\right)_{\nu \geq 0}$ be a sequence of complex numbers and $S_{n}(z)=\sum_{\nu=0}^{n} c_{\nu} z^{\nu}$ the associated Taylor series. Set $S_{n}=S_{n}(1)$. Suppose that the series $\sum_{\nu>0} c_{\nu}$ is $(C, 1)$ summable to $\sigma \in \mathbb{C}$. Let $\mathcal{D}$ be an infinite subset of $\mathbb{N}$, and for every $n \in \mathcal{D}$, let $z_{n}$ be a complex number such that $\lim _{n \rightarrow+\infty, n \in \mathcal{D}} n\left(1-z_{n}\right)=u \neq 0$. Then

$$
\lim _{n \rightarrow+\infty, n \in \mathcal{D}} z_{n}^{-n}\left(S_{n}\left(z_{n}\right)-\sigma\right)-\left(S_{n}-\sigma\right)=0 .
$$

The proof of Theorem 3.2 is based on the following claim: 
Claim. There exists a residual set $G \subset \mathbb{T}$ such that for every boundary point $w \in G$, and every open disk $D_{w} \subset \mathbb{D}$ with $\mathbb{T} \cap \overline{D_{w}}=\{w\}$, the set $f\left(D_{w}\right)$ is unbounded.

We shall actually prove that there is at most one point $w \in \mathbb{T}$ such that there exists a disk $D_{w} \subset \mathbb{D}$ with $\mathbb{T} \cap \overline{D_{w}}=\{w\}$ and $f\left(D_{w}\right)$ bounded. Suppose to the contrary that there are two such points. Without loss of generality, one may assume that one of them is 1 , and denote the second one by $w . D_{1}$ and $D_{w}$ will stand for the disks where $f$ is bounded, and $\xi_{1}\left(\operatorname{resp} . \xi_{w}\right)$ is the center of $D_{1}$ (resp. $\left.D_{w}\right)$. Let $F(z)=\int_{(0, z)} f(u) d u$, where $(0, z)$ is any path $($ in $\mathbb{D})$ connecting 0 to $z \in \mathbb{D}$. Since $F^{\prime}=f$ is bounded on $D_{1}, F$ has a continuous extension to $\overline{D_{1}}$. By Fejer's Theorem, there exists $\ell_{1} \in \mathbb{C}$ such that $\sigma_{n}^{1}\left(F, \xi_{1}\right)(1) \rightarrow \ell_{1}$ as $n \rightarrow \infty$. Analogously, there is $\ell_{w} \in \mathbb{C}$ such that $\sigma_{n}^{1}\left(F, \xi_{w}\right)(w) \rightarrow \ell_{w}$ as $n \rightarrow \infty$.

Let $\Gamma$ be a closed arc on $\mathbb{T}$ from $w$ to 1 , and take $P$ any holomorphic polynomial such that $\int_{\Gamma} P(z) d z \neq \ell_{1}-\ell_{w}$. We denote by $C$ the compact subset of $\mathbb{C}$ defined by $C=\left\{r e^{i \theta} ; e^{i \theta} \in \Gamma, 1 \leq r \leq 2\right\}$. By Lemma 2.3, there exist two sequences of natural numbers $\left(p_{m}\right),\left(q_{m}\right)$ such that the Taylor series of $f$ with center at $\xi_{w}$ has Ostrowski gaps $\left(p_{m}-1, q_{m}\right)$, and

$$
\sup _{z \in C}\left|S_{p_{m}-1}\left(f, \xi_{w}\right)(z)-P(z)\right| \rightarrow 0, \quad \text { as } \quad m \rightarrow+\infty .
$$

Since $F$ is obtained by integration termwise of the power series of $f$, its power series with center $\xi_{w}$ has Ostrowski gaps $\left(p_{m}, q_{m}\right)$. Writing $S_{n}\left(f, \xi_{w}\right)(z)=\sum_{\nu=0}^{n} a_{\nu}\left(z-\xi_{w}\right)^{\nu}$, so that $S_{n}\left(F, \xi_{w}\right)(z)=F\left(\xi_{w}\right)+\sum_{\nu=0}^{n-1} \frac{a_{\nu}}{\nu+1}\left(z-\xi_{w}\right)^{\nu+1}$, we have

$$
\begin{aligned}
\sigma_{n}^{1}\left(F, \xi_{w}\right)(z)= & F\left(\xi_{w}\right)+a_{0}\left(z-\xi_{w}\right)\left(1-\frac{1}{n+1}\right)+\frac{a_{1}}{2}\left(z-\xi_{w}\right)^{2}\left(1-\frac{2}{n+1}\right) \\
& +\cdots+\frac{a_{n-1}}{n}\left(z-\xi_{w}\right)^{n}\left(1-\frac{n}{n+1}\right) \\
= & S_{n}\left(F, \xi_{w}\right)(z)-\frac{\left(z-\xi_{w}\right)}{n+1} S_{n-1}\left(f, \xi_{w}\right)(z) .
\end{aligned}
$$

We apply this formula for $n=p_{m}$ and $z=w$, and we let $m$ go to infinity. Since the sequence $\left(S_{p_{m}-1}\left(f, \xi_{w}\right)(w)\right)$ is bounded, the sequence $\left(S_{p_{m}}\left(F, \xi_{w}\right)(w)\right)$ converges to $\ell_{w}$. Integrating along $\Gamma$, we deduce that

$$
S_{p_{m}}\left(F, \xi_{w}\right)(1) \stackrel{m \rightarrow+\infty}{\longrightarrow} \ell_{w}+\int_{\Gamma} P:=\alpha .
$$

On the other hand, Lemma 2.2 ensures that $S_{p_{m}}\left(F, \xi_{1}\right)(1)$ tends also to $\alpha$. We write $S_{n}\left(F, \xi_{1}\right)(z)=\sum_{\nu=0}^{n} b_{\nu}\left(z-\xi_{1}\right)^{\nu}$ and set $z_{p_{m}}=1+\frac{1}{p_{m}}$. In order to apply Rogosinski's Formula, it is convenient to set $c_{\nu}=b_{\nu}\left(1-\xi_{1}\right)^{\nu}$. The series $\sum_{\nu>0} c_{\nu}$ is $(C, 1)$-summable, and in the notation of Lemma 3.3, one has $S_{n}=S_{n}\left(F, \xi_{1}\right)(1)$ and 
$S_{p_{m}}\left(z_{p_{m}}\right)=S_{p_{m}}\left(F, \xi_{1}\right)\left(1+\frac{1}{p_{m}}-\frac{\xi_{1}}{p_{m}}\right)$. By uniform convergence on $C, S_{p_{m}}\left(z_{p_{m}}\right)$ tends to $\alpha$. So, Lemma 3.3 gives

$$
\lim _{m \rightarrow+\infty} z_{p_{m}}^{-p_{m}}\left(\alpha-\ell_{1}\right)+\left(\alpha-\ell_{1}\right)=0 .
$$

Observe now that $\lim _{m \rightarrow+\infty} z_{p_{m}}^{-p_{m}}=e^{-1}$ to deduce that $\alpha=\ell_{1}$ : this contradicts $\int_{\Gamma} P \neq \ell_{1}-\ell_{w}$. Thus, the claim is proved.

Theorem 3.2 can now be deduced from the claim as in [1]. For the sake of completeness, we shall include some details. The radial cluster set $C_{\rho}(f, z)$ of $f$ at $z$ is defined in the following way: $a \in C_{\rho}(f, z)$ if there exists a sequence $\left(r_{n}\right) \in(0,1)$ such that $\lim _{n \rightarrow+\infty} r_{n}=1$ and $\lim _{n \rightarrow+\infty} f\left(r_{n} z\right)=a$. The cluster set $C(f, z)$ of $f$ at $z$ consists of all points $a$ for which there exists a sequence $\left(z_{n}\right) \in \mathbb{D}$ having the properties $\lim _{n \rightarrow \infty} z_{n}=z$ and $\lim _{n \rightarrow \infty} f\left(z_{n}\right)=a$. Our claim shows that for every $z$ in a residual subset $G \subset \mathbb{T}, C(f, z)$ is unbounded. We conclude by Collingwood's maximality Theorem (see [3]) which asserts that for a residual set of points $z$ on the unit circle, $C_{\rho}(f, z)=C(f, z)$.

\section{Cesàro means of universal Taylor series}

We begin this section by two lemmas. The first one is an elementary geometrical lemma.

Lemma 4.1. Let $K$ be a compact subset of $\mathbb{C}$ such that $\mathbb{C} \backslash K$ is connected and $K \cap \mathbb{D}=\emptyset$. Then there exist two compact subsets $L$ and $M$ of $\mathbb{C}$ and a positive real number $\alpha>0$ such that

- $L \cap \mathbb{D}=M \cap \mathbb{D}=\emptyset, K \subset L \subset M$.

- $\mathbb{C} \backslash M$ is connected.

- For any $z \in L$, there exists $w \in L$ such that $z \in \bar{D}(w, \alpha)$ and $\bar{D}(w, \alpha) \subset L$.

Proof. Let $A \geq 1$ be an integer such that $K \subset\{z \in \mathbb{C} ; 1 \leq|z| \leq A\}$. Since 0 and $A+1$ belong to the complement of $K$, which is connected, we can join them by a simple polygonal line $\Gamma$ lying in the complement of $K$. The distance of $K$ to $\Gamma$ is positive, greater than or equal to $3 \alpha, 0<\alpha \leq \frac{1}{2}$. We then set:

$$
\begin{gathered}
M=\{z \in \mathbb{C} ; 1 \leq|z| \leq A+2 \alpha, \operatorname{dist}(z, \Gamma) \geq \alpha\}, \\
L_{0}=\{w \in \mathbb{C} ; 1+\alpha \leq|w| \leq A+\alpha, \operatorname{dist}(w, \Gamma) \geq 2 \alpha\}, \\
L=\bigcup_{w \in L_{0}} \bar{D}(w, \alpha)=\left\{z \in \mathbb{C} ; \operatorname{dist}\left(z, L_{0}\right) \leq \alpha\right\} .
\end{gathered}
$$

One can easily check that $M, L_{0}$, and $L$ are compact subsets of $\mathbb{C}$, that $M \cap \mathbb{D}=$ $L \cap \mathbb{D}=\emptyset$, that $\mathbb{C} \backslash M$ is connected, and that $L \subset M$. (For the last assertion, observe 
that for $z$ in $L, \gamma$ in $\Gamma$ and $w$ in $L_{0}$ with $z \in \bar{D}(w, \alpha)$, the following inequalities are satisfied: $|z-\gamma| \geq|w-\gamma|-|z-w| \geq 2 \alpha-\alpha \geq \alpha$.) Moreover, by construction of $L$, for any $z \in L$, there exists $w \in L$ such that $z \in \bar{D}(w, \alpha)$ and $\bar{D}(w, \alpha) \subset L$. It remains to prove that $K$ is contained in $L$. Take any $z \in K$, and set $w=z+\frac{z}{|z|} \alpha$. It is straightforward that $1+\alpha \leq|w| \leq A+\alpha$. On the other hand, take any $\gamma \in \Gamma$. One has $|w-\gamma| \geq|z-\gamma|-\alpha \geq 2 \alpha$. This proves that $w$ belongs to $L_{0}$, and since $z$ lies in $\bar{D}(w, \alpha)$, one gets $z \in L$.

We will also need a classical lemma which comes from Harmonic Analysis.

Lemma 4.2. Let $P$ be a holomorphic polynomial of degree $N, w \in \mathbb{C}, \alpha>0, k \geq 1$. Then one has

$$
\sup _{z \in \bar{D}(w, \alpha)}\left|P^{(k)}(z)\right| \leq \frac{N(N-1) \cdots(N-k+1)}{\alpha^{k}} \sup _{z \in \bar{D}(w, \alpha)}|P(z)| .
$$

Proof. For $w=0$ and $\alpha=1$, this is just Bernstein's inequality (see for instance [6]). Otherwise, we set $Q(z)=P(\alpha z+w)$, so that

$$
\sup _{z \in \bar{D}(0,1)}\left|Q^{(k)}(z)\right|=\alpha^{k} \sup _{z \in \bar{D}(w, \alpha)}\left|P^{(k)}(z)\right| .
$$

Theorem 4.3. Let $f \in H(\mathbb{D})$, and $\xi_{0} \in \mathbb{D}$. Suppose that there exists $k \geq 0$ such that, for any compact set $K \subset \mathbb{C} \backslash \mathbb{D}$, with $K^{c}$ connected and for every function $h$ continuous on $K$ and holomorphic in $K^{0}$, there exists a sequence $\left(\lambda_{m}\right)$ of natural numbers such that

$$
\sup _{z \in K}\left|\sigma_{\lambda_{m}}^{k}\left(f, \xi_{0}\right)(z)-h(z)\right| \rightarrow 0 \quad \text { as } \quad m \rightarrow+\infty .
$$

Then for any compact set $K \subset \mathbb{C} \backslash \mathbb{D}$ with $K^{c}$ connected and for any holomorphic polynomial $P$, there exists a sequence $\left(\lambda_{m}\right)$ of natural numbers such that, for any compact subset $N$ of $\mathbb{D}$, for every $j \in \mathbb{N}$,

$$
\sup _{\xi \in N} \sup _{z \in K}\left|\sigma_{\lambda_{m}}^{j}(f, \xi)(z)-P(z)\right| \rightarrow 0 \quad \text { as } \quad m \rightarrow+\infty .
$$

Proof. We will extend formula (1) to $\operatorname{link} \sigma_{n}^{j}(f, \xi)(z)$ to $S_{n}(f, \xi)(z)$ and its derivatives. Writing $S_{n}(f, \xi)(z)=\sum_{\nu=0}^{n} a_{\nu}(z-\xi)^{\nu}$, we have

$$
\begin{aligned}
\sigma_{n}^{j}(f, \xi)(z)= & S_{n}(f, \xi)(z) \\
& +\sum_{\nu=0}^{n} \frac{[(n+1)-\nu] \cdots[(n+j)-\nu]-(n+1) \cdots(n+j)}{(n+1) \cdots(n+j)} a_{\nu}(z-\xi)^{\nu} \\
= & S_{n}(f, \xi)(z) \\
& +\sum_{\nu=0}^{n} \frac{P_{j}(n) \nu(\nu-1) \cdots(\nu-j+1)+\cdots+P_{1}(n) \nu}{(n+1) \cdots(n+j)} a_{\nu}(z-\xi)^{\nu},
\end{aligned}
$$


where each $P_{l}$ is a polynomial (whose coefficients depend only on $j$ ) with $\operatorname{deg}\left(P_{l}\right) \leq$ $j-l$. This in turn gives

$$
\begin{aligned}
\sigma_{n}^{j}(f, \xi)(z)=S_{n}(f, \xi)(z)+Q_{j}(n)(z-\xi)^{j} S_{n-j}\left(f^{(j)}, \xi\right)(z) & +\cdots \\
& +Q_{1}(n)(z-\xi) S_{n-1}\left(f^{\prime}, \xi\right)(z)
\end{aligned}
$$

where $Q_{l}$ is a rational function such that the difference of the degrees of numerator and denominator is less than or equal to $-l$. The proof will be done in two steps. First, we show that the statement of Theorem 4.3 is true for $k=0$, i.e., if $f$ belongs to $U\left(\mathbb{D}, \xi_{0}\right)$. As recalled in the introduction, we have in fact $f \in U(\mathbb{D})$. So, let $K \subset \mathbb{C} \backslash \mathbb{D}$ be a compact set such that $K^{c}$ is connected, and let $P$ be a holomorphic polynomial. We define $L$ and $M$ as the compact subsets of $\mathbb{C}$ given by Lemma 4.1, and $\alpha>0$ is the positive number associated with these data. By Lemma 2.3, there exist two sequences of natural numbers $\left(p_{m}\right)$ and $\left(q_{m}\right)$ so that the Taylor series of $f$ at center $\xi_{0}$ has Ostrowski-gaps $\left(p_{m}, q_{m}\right)$ and for any compact set $N \subset \mathbb{D}$,

$$
\sup _{\xi \in N} \sup _{z \in M}\left|S_{p_{m}}(f, \xi)(z)-P(z)\right| \rightarrow 0 \quad \text { as } \quad m \rightarrow+\infty .
$$

Hence, if we fix a compact set $N \subset \mathbb{D}$, there exists a constant $C_{1} \in(0,+\infty)$ such that, for any $m \geq 0$, we have

$$
\sup _{\xi \in N} \sup _{w \in L}\left|S_{p_{m}}(f, \xi)(w)\right| \leq C_{1}
$$

Pick any $z \in K$ and $\xi \in N$. There exists $w \in L$ such that $\bar{D}(w, \alpha) \subset L$ and $z \in \bar{D}(w, \alpha)$. By Lemma 4.2, one obtains

$$
\left|S_{p_{m}-l}\left(f^{(l)}, \xi\right)(z)\right| \leq \frac{p_{m} \cdots\left(p_{m}-l+1\right)}{\alpha^{l}} C_{1}
$$

We now consider a sequence $\left(r_{m}\right)$ of natural numbers such that $r_{m} / p_{m} \rightarrow+\infty$ and $q_{m} / r_{m} \rightarrow+\infty$. (For instance, $r_{m}=\left[p_{m}^{1 / 2} q_{m}^{1 / 2}\right]$ will do.) By Lemma 2.4, there exists another constant $C_{2}$ such that for $z \in K, l \in\{1, \ldots, j\}$ and $m \geq 0$, one has

$$
\sup _{\xi \in N} \sup _{z \in K}\left|S_{r_{m}-l}\left(f^{(l)}, \xi\right)(z)\right| \leq \sup _{\xi \in N} \sup _{z \in K}\left|S_{p_{m}-l}\left(f^{(l)}, \xi\right)(z)\right|+C_{2} .
$$

Putting this together, and using the fact that $p_{m} / r_{m}$ goes to 0 , one has proved that for any $l$ in $\{1, \ldots, j\}, Q_{l}\left(r_{m}\right)(z-\xi)^{l} S_{r_{m}-l}\left(f^{(l)}, \xi\right)(z)$ tends to 0 uniformly on $N \times K$ $(\xi \in N, z \in K)$. Therefore, if we apply equation (2) for $n=r_{m}$, we get that $\sigma_{r_{m}}^{j}(f, \xi)(z)-P(z)$ converges to 0 uniformly on $N \times K$, and this completes the first part of the proof of Theorem 4.3.

Secondly, suppose that $f$ satisfies the assumptions of Theorem 4.3 for a given $k \geq 1$. Let $K$ be any compact subset of $\mathbb{C} \backslash \mathbb{D}$ with $K^{c}$ connected, and let $P$ be any 
holomorphic polynomial. We are going to prove that there exists a sequence $\left(\lambda_{m}\right)$ of natural numbers such that $S_{\lambda_{m}}\left(f, \xi_{0}\right)(z)$ converges uniformly to $P$ on $K$. By the first part of the proof, this is clearly sufficient to obtain Theorem 4.3. The proof is again based on identity (2). Let $K$ and $P$ be defined as above and consider $L, M$, and $\alpha>0$ given by Lemma 4.1. Lemma 2.6 gives two sequences of natural numbers $\left(p_{m}\right)$ and $\left(q_{m}\right)$ such that the Taylor series of $f$ at center $\xi_{0}$ has Ostrowski-gaps $\left(p_{m}, q_{m}\right)$ and

$$
\sigma_{q_{m}}^{k}\left(f, \xi_{0}\right)(z) \rightarrow P(z) \quad \text { uniformly on } M .
$$

Let $\varepsilon_{m}=\sup _{z \in L}\left|S_{q_{m}}\left(f, \xi_{0}\right)(z)-P(z)\right|$, and let $C_{3}=\sup _{z \in L}|P(z)|$. (Observe that we do not know a priori whether $\varepsilon_{m}$ goes to 0 .) Since for any $z \in L$, there exists $w \in L$ such that $\bar{D}(w, \alpha) \subset L$, Lemma 4.2 ensures that for $l \in\{0, \ldots, k\}$, we have

$$
\sup _{z \in L}\left|S_{p_{m}-l}\left(f^{(l)}, \xi_{0}\right)(z)\right| \leq \frac{p_{m} \cdots\left(p_{m}+1-l\right)}{\alpha^{l}} \sup _{z \in L}\left|S_{p_{m}}\left(f, \xi_{0}\right)(z)\right| .
$$

Writing

$$
S_{q_{m}-l}\left(f^{(l)}, \xi_{0}\right)=S_{q_{m}-l}\left(f^{(l)}, \xi_{0}\right)-S_{p_{m}-l}\left(f^{(l)}, \xi_{0}\right)+S_{p_{m}-l}\left(f^{(l)}, \xi_{0}\right)
$$

and

$$
S_{p_{m}}\left(f, \xi_{0}\right)=S_{p_{m}}\left(f, \xi_{0}\right)-S_{q_{m}}\left(f, \xi_{0}\right)+S_{q_{m}}\left(f, \xi_{0}\right)-P+P
$$

and denoting $\varepsilon_{m}^{(l)}=\sup _{z \in L}\left|S_{p_{m}-l}\left(f^{(l)}, \xi_{0}\right)(z)-S_{q_{m}-l}\left(f^{(l)}, \xi_{0}\right)(z)\right|$ (which clearly tends to 0 by the definition of Ostrowski gaps), one deduces

$$
\sup _{z \in L}\left|S_{q_{m}-l}\left(f^{(l)}, \xi_{0}\right)(z)\right| \leq \varepsilon_{m}^{(l)}+\frac{p_{m} \cdots\left(p_{m}+1-l\right)}{\alpha^{l}}\left(\varepsilon_{m}^{(0)}+\varepsilon_{m}+C_{3}\right) .
$$

Suppose now $l \geq 1$. Recall that the difference of the degrees of the numerator and the denominator of $Q_{l}$ is less than or equal to $-l$. Hence, since $p_{m} \cdots\left(p_{m}+1-l\right) \leq p_{m}^{l}$ and since $p_{m} \leq q_{m}$, the boundedness of $\left(\varepsilon_{m}^{(l)}\right)_{m}$ and of $\left(\varepsilon_{m}^{(0)}\right)_{m}$ yields the existence of a constant $D_{l}$ independent on $m$, such that

$$
\sup _{z \in L}\left|Q_{l}\left(q_{m}\right)\right|\left|z-\xi_{0}\right|^{l}\left|S_{q_{m}-l}\left(f^{(l)}, \xi_{0}\right)(z)\right| \leq D_{l} \frac{p_{m}}{q_{m}}\left(1+\varepsilon_{m}\right),
$$

Using (2) we obtain

$$
\varepsilon_{m} \leq \sup _{z \in L}\left|\sigma_{q_{m}}^{(k)}\left(f, \xi_{0}\right)(z)-P(z)\right|+\left(D_{1}+\cdots+D_{k}\right) \frac{p_{m}}{q_{m}}\left(1+\varepsilon_{m}\right),
$$

which in turn gives

$$
\varepsilon_{m}\left(1-\left(D_{1}+\cdots+D_{k}\right) \frac{p_{m}}{q_{m}}\right) \leq \sup _{z \in L}\left|\sigma_{q_{m}}^{(k)}\left(f, \xi_{0}\right)(z)-P(z)\right|+\left(D_{1}+\cdots+D_{k}\right) \frac{p_{m}}{q_{m}} .
$$

Since $p_{m} / q_{m} \rightarrow 0$ as $m \rightarrow+\infty$, we get that $\varepsilon_{m}$ tends to 0 , and Theorem 4.3 is proved. 
Corollary 4.4. For any $\xi \in \mathbb{D}$ and any $k \geq 0$, we have the following equalities:

$$
U(\mathbb{D})=U_{\mathrm{Ces}}(\mathbb{D})=U_{\mathrm{Ces}}(\mathbb{D}, \xi)=U_{\mathrm{Ces}(k)}(\mathbb{D})=U_{\mathrm{Ces}(k)}(\mathbb{D}, \xi) .
$$

Proof. We just prove that $U_{\mathrm{Ces}(\mathbb{D})}=U(\mathbb{D})$; the remaining identities are proved in the same way. Let $f \in U(\mathbb{D})$, let $K$ be a compact subset of $\mathbb{C}, K \cap \mathbb{D}=\emptyset, K^{c}$ connected, and let $h: K \rightarrow \mathbb{C}$ be a continuous function on $K$ and holomorphic in $K^{0}$. By Mergelyan's Theorem, there exists a sequence $\left(P_{l}\right)$ of holomorphic polynomials such that $P_{l} \rightarrow h$ uniformly on $K$. For any $l \geq 1$, there exists a sequence $\left(\lambda_{m}(l)\right)_{m \geq 0}$ such that, for any $k \geq 0$ and any compact set $N \subset \mathbb{D}$, one has

$$
\sup _{z \in K}\left|\sigma_{\lambda_{m}(l)}^{k}\left(f, \xi_{0}\right)(z)-P_{l}(z)\right| \rightarrow 0 \quad \text { as } \quad m \rightarrow+\infty .
$$

Using a diagonal argument, we easily deduce Corollary 4.4.

Remark 4.5. The previous theorem gives in particular another proof (without using Rogosinski's formula) that the Taylor expansion of $f \in U(\mathbb{D})$ with center at $\xi \in \mathbb{D}$ cannot be $(C, k)$-summable at any point of $\mathbb{T}$.

Remark 4.6. In Theorem 4.3, the (arbitrary) polynomial $P$ may be replaced by any entire function. The proof is unchanged.

Remark 4.7. Melas and Nestoridis' results, and the results about Ostrowski gaps of Taylor series, are also valid if one replaces $\mathbb{D}$ by a simply connected domain $\Omega$ contained in some half-plane. On the other hand, our method of proof requires the geometrical Lemma 4.1. So, our result is still valid if we replace $\mathbb{D}$ by another simply connected domain contained in some half-plane for which Lemma 4.1 remains true (for instance, a square). We do not know what happens for domains where Lemma 4.1 does not hold. In the same vein, we do not know if our result is still valid if we consider $(C, a)$-means, with $a \in(-1,+\infty)$ not necessarily a natural number.

\section{References}

[1] D. Armitage and G. Costakis, Boundary behavior of universal Taylor series and their derivatives, Constr. Approx., to appear.

[2] C. K. Chui and M. N. Parnes, Approximation by overconvergence of a power series, J. Math. Anal. Appl. 36 (1971), 693-696.

[3] E. F. Collingwood and A. J. Lohwater, The theory of cluster sets, Cambridge Tracts in Mathematics and Mathematical Physics, vol. 56, Cambridge University Press, Cambridge, 1966.

[4] G. Costakis, On the radial behavior of universal Taylor series, Monatsh. Math. 145 (2005), no. $1,11-17$.

[5] W. Gehlen, W. Luh, and J. Müller, On the existence of O-universal functions, Complex Variables Theory Appl. 41 (2000), no. 1, 81-90.

[6] J.-P. Kahane, Some random series of functions, Cambridge Studies in Advanced Mathematics, vol. 5, Cambridge University Press, Cambridge, 1985. 
[7] E. S. Katsoprinakis and M. Papadimitrakis, Extensions of a theorem of Marcinkiewicz-Zygmund and of Rogosinski's formula and an application to universal Taylor series, Proc. Amer. Math. Soc. 127 (1999), no. 7, 2083-2090.

[8] W. Luh, Approximation analytischer Funktionen durch überkonvergente Potenzreihen und deren Matrix-Transformierten, Mitt. Math. Sem. Giessen Heft 88 (1970), i+56.

[9]__ Universal approximation properties of overconvergent power series on open sets, Analysis 6 (1986), no. 2-3, 191-207.

[10] A. Melas and V. Nestoridis, Universality of Taylor series as a generic property of holomorphic functions, Adv. Math. 157 (2001), no. 2, 138-176.

[11] V. Nestoridis, Universal Taylor series, Ann. Inst. Fourier (Grenoble) 46 (1996), no. 5, 1293-1306.

[12] J. L. Walsh, Interpolation and approximation by rational functions in the complex domain, 3rd edition, American Mathematical Society Colloquium Publications, vol. XX, American Mathematical Society, Providence, R.I., 1960.

[13] A. Zygmund, Trigonometric series, 2nd edition, American Mathematical Society Colloquium Publications, Cambridge University Press, Cambridge, 1979. 